\title{
Should the Pendulum Swing Back? More Transfers to the ICU After Implementing Ward-Based High-Flow Nasal Cannula Initiation Protocols for Bronchiolitis
}

\author{
Daniel J Sklansky, MD*, Sanjay Mahant, MD, FRCPC, MSc²
}

'Division of Hospital Medicine, Department of Pediatrics, University of Wisconsin School of Medicine and Public Health, Madison, Wisconsin; ${ }^{2}$ Division of Pediatric Medicine, Department of Pediatrics and Institute of Health Policy, Management and Evaluation, University of Toronto, Toronto, Canada.

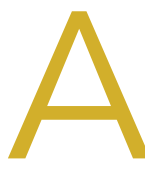

s an appealing, physiologically plausible treatment, humidified oxygen delivery via high-flow nasal cannula (HFNC) has been rapidly adopted for the treatment of bronchiolitis despite weak evidence supporting its routine and early use in hypoxemic infants. ${ }^{1} \mathrm{Al}-$ though HFNC use has been associated with decreased work of breathing and lower rates of progression to invasive ventilation in some studies, the one large trial published on the topic found no difference between early HFNC and standard oxygen therapy on length of stay in hospital, duration of oxygen therapy, or rates of intubation. ${ }^{2,3} \mathrm{No}$ adequately powered studies have examined the effect of ward-based HFNC initiation on ICU transfer, an outcome that it is designed to prevent.

In this month's issue of the Journal of Hospital Medicine, Coon et al examine the association between the implementation of ward-based HFNC initiation protocols and subsequent ICU transfer rates. ${ }^{4}$ Hospitals enrolled in the Pediatric Health Information System database were surveyed about their HFNC use and protocol implementation, with 41 (93\% response rate) hospitals replying, 12 of which implemented ward-based HFNC initiation protocols during 2010 to 2016. Administrative data for bronchiolitis encounters were obtained with use of International Classification of Diseases, 9th and 10th Revisions, coding of children aged 3 to 24 months discharged during the respiratory seasons of the study period. The authors used an interrupted time series analysis to study the association between ward-based HFNC protocol initiation and several outcomes, revealing a small but significant increase in ICU transfers (absolute difference, 3.1\%; 95\% Cl, 2.8\%-3.4\%) and ICU length of stay (absolute difference, 9.1 days per 100 patients; $95 \% \mathrm{Cl} 5.1-13.2)$, but not overall length of stay or use of mechanical ventilation. Modifications to the analysis that account for a learning period during the first season of implementation at each hospital, and for trends among nonadopting hospitals, did not substantially affect the findings.

The authors acknowledged many of the study's limitations, including its retrospective design, presumption of bronchiolitis discharge code validity, restriction to tertiary care hospitals, and analysis of hospital-level rather than patient-level vari-

"Corresponding Author: Daniel J Sklansky, MD; Email: djsklansky@pediatrics. wisc.edu; Telephone: 608-262-5626.

Received: May 5, 2020; Revised: May 6, 2020; Accepted: May 6, 2020

(๑) 2020 Society of Hospital Medicine DOI 10.12788/jhm.3462 ables and outcomes. Because the data source does not capture patient-level HFNC use, the number and characteristics of patients receiving HFNC at the centers are unknown. It is also important to note that the 12 included protocols are quite heterogeneous, with differing exclusion criteria, maximum flow rates, and indications for ICU transfer. Given the rapid evolution of ward-based HFNC use for bronchiolitis, these protocols from 2010 to 2016 are already out of date. All of the protocols allowed much lower maximum flow rates (4-10 L/min) than would typically be expected today (usually $2 \mathrm{~L} / \mathrm{kg}$ per minute, which translates to $10 \mathrm{~L} / \mathrm{min}$ of flow for a $5-\mathrm{kg}$ child or $20 \mathrm{~L} / \mathrm{min}$ for a $10-k g$ child). Many also had time-based criteria prompting ICU transfer (eg, 24 hours without improvement) that are not typically included in more recent protocols. Few had instructions for weaning or discontinuation of HFNC.

In spite of the above limitations, the results of this large, multicenter study advance our understanding of the consequences of ward-based protocols for HFNC initiation. However, it is important to contextualize this work as an examination of the implementation of a technology to a broad population in a specific era, not necessarily a study of the effectiveness of the technology itself.

The pediatric hospital medicine community has long recognized the need for more evidence regarding HFNC use. ${ }^{5-7}$ Coon et al have highlighted possible unintended consequences, notably increased ICU use, that may be associated with ward-based HFNC implementation on a population basis. This finding mirrors evidence from a recent similarly designed study analyzing Canadian tertiary care centers implementing HFNC administration during 2009 to 2014, though not specifically limited to ward use. ${ }^{8}$

More recently there has been discussion of how we might deimplement ward-based HFNC protocols. Although it is increasingly clear that HFNC is not a panacea for bronchiolitis, there is not necessarily a problem with the technology; the problem that this study so clearly demonstrates is how we have applied it. We need pragmatic trials of HFNC protocols to understand what parameters should guide HFNC initiation as a rescue treatment; what oxygen and flow settings might prevent ICU transfer; how it should be used in populations that have been largely excluded from trials (ie, children with medical complexity); and how to optimally wean it. With that information we could construct evidence-based, utilitarian HFNC initiation and treatment protocols to maximize benefit and minimize harm and cost. 
It is understandable that our desire to help patients has led us to hear the "siren's call" for this therapy, and indeed we should work on putting some of the "horses back in the barn. "5,6 Until new evidence guides how to best use this technology, institutional practice guidelines for HFNC initiation in ward settings should target children for whom ICU transfer seems very likely (eg, having oxygen saturations not maintained on maximum low-flow oxygen therapy) so that HFNC is not used routinely and that we maximize its cost to benefit ratio. It is important to approach this shift in a thoughtful manner to prevent a pendulum swing to premature universal deimplementation.

Disclosures: The authors have nothing to disclose.

\section{References}

1. Piper L, Stalets EL, Statile AM. Clinical practice update: high flow nasal cannula therapy for bronchiolitis outside the ICU in infants. J Hosp Med.
2019;14:E1-E3. https://doi.org/10.12788/jhm.3328.

2. Franklin $\mathrm{D}, \mathrm{Bab}$ FE, Schlapbach LJ, et al. A randomized trial of high-flow oxygen therapy in infants with bronchiolitis. N Engl J Med. 2018;378(12):11211131. https://doi.org/10.1056/nejmoa1714855.

3. Lin J, Zhang Y, Xiong L, Liu S, Gong C, Dai J. High-flow nasal cannula therapy for children with bronchiolitis: a systematic review and meta-analysis. Arch Dis Child. 2019;104(6):564-576. https://doi.org/10.1136/archdischild-2018-315846.

4. Coon ER, G. S, Brady PW. Intensive care unit utilization after adoption of a ward-based high-flow nasal cannula protocol. J Hosp Med. 2020;15(6):325330. https://doi.org/10.12788/jhm.3456.

5. de Benedictis FM. The Effectiveness of high-flow oxygen therapy and the fascinating song of the sirens. JAMA Pediatr. 2019;173(2):125-126. https:// doi.org/10.1001/jamapediatrics.2018.3831.

6. Ralston SL. High-flow nasal cannula therapy for pediatric patients with bronchiolitis: time to put the horse back in the barn [online first]. JAMA Pediatr. 2020. https://doi.org/10.1001/jamapediatrics.2020.0040.

7. Ralston SL, Lieberthal AS, Meissner HC, et al. Clinical practice guideline: the diagnosis, management, and prevention of bronchiolitis. Pediatrics. 2014;134(5):e1474-e1502. https://doi.org/10.1542/peds.2015-2862.

8. Garland H, Miller MR, Gunz AC, Lim RK. High-flow nasal cannula implementation has not reduced intubation rates for bronchiolitis in Canada [online first]. Paediatr Child Health. 2020. https://doi.org/10.1093/pch/pxaa023. 